\title{
Prevalence of Third Molar Agenesis in Patients - A Panoramic Retrospective Study
}

\author{
Vivek Padmanabhan, Omar Khaled AR Abo Mostafa and Lama M. Kamel Rahhal
}

\section{ABSTRACT}

Background/Purpose: Agenesis is found to be the most common dental anomaly affecting at least $25 \%$ of the population. Agenesis is an anomaly where the tooth germ fails to differentiate completely into dental tissues resulting in congenitally missing teeth. The aim of this study was to determine the prevalence of bilateral agenesis of mandibular second premolars. This study also reflects upon the treatment options available for the agenesis of mandibular second premolars.

Materials and Methods: Bilateral agenesis was considered as agenesis and unilateral agenesis was excluded from the study. Orthopantamograms (OPGs) of 945 dental patients aged 6 years $\mathbf{- 3 0}$ years were examined for the agenesis of teeth. Descriptive statistics were used to describe the percentages and frequencies were calculated using chi square test and the level of significance was considered if $p$ value was $<0.05$.

Results: The prevalence of bilateral agenesis or congenitally missing mandibular second premolars were at $\mathbf{1 0 . 5 \%}$.

Conclusions: The prevalence rates of bilateral agenesis or congenitally missing mandibular second premolars are more common in males than females. A well informed evidence based decision should be taken for the clinical management of the missing mandibular second premolars.

Keywords: Mandibular Second Premolars, OPG, Bilateral Agenesis.
Published Online: March 25, 2021

ISSN: 2684-4443

DOI: $10.24018 /$ ejdent.2021.2.2.44

\section{Vivek Padmanabhan*}

Assistant Professor

Pediatric and Preventive Dentistry, RAK College of Dental Sciences, RAK Medical and Health Sciences University, Ras Al Khaimah, United Arab Emirates.

(e-mail: vivek.padmanabhan ${ }^{\circledR}$ rakmhsu.ac.ae) Mr Omar Khaled AR Abo Mostafa 5th Year BDS Student

RAK College of Dental Sciences, RAK Medical and Health Sciences University, Ras Al Khaimah, United Arab Emirates.

Ms Lama M. Kamel Rahhal 5th Year BDS Student

RAK College of Dental Sciences RAK Medical and Health Sciences University, Ras Al Khaimah, United Arab Emirates.

*Corresponding Author

\section{INTRODUCTION}

Several terms are used to reflect upon different types of agenesis of teeth. Agenesis occurs when the tooth germs fail to differentiate appropriately into dental tissues [1], [2]. One of the commonest dental anomalies reported is agenesis with a prevalence rate of $25 \%$ approximately amongst the general population [3], [4]. There are different terms used to describe variants of agenesis. Agenesis when seen in less than six teeth (excluding third molars) is defined as Hypodontia [4]. The term Oligodontia is defined as the condition when there is agenesis of six or more teeth and the term Anodontia refers to condition when there is complete agenesis of teeth [5], [6].

Agenesis excluding the third molars is commonly seen in the mandibular second premolars followed by the maxillary lateral incisors as reported in various researches [7], [8]. As a consequence of missing teeth in the premolar or anterior region, there could be occurrence of discrepancies in arch length, unaesthetic appearance and malocclusion [9], [10].

There are different factors which have been suggested as reasons for agenesis and these include evolution of humans, masticatory apparatus changes, dietary factors, and genetics [11], [12].
When races have been compared it has been seen that there is an increased prevalence of agenesis amongst the whites and Asians as compared to Non-Asians and black race [13]. Agenesis is seen in both the permanent and primary dentition with a higher predilection for the permanent dentition when compared to the primary dentition [13].

Orthodontic treatments are most affected when there is agenesis of premolars. The diagnosis of agenesis of premolars is impeded by calcification of these teeth which is delayed. The inability to determine agenesis at an early stage can result in a space loss or also collapse of the dental arch [12], [13].

When the agenesis or congenitally missing teeth is in the functional, esthetic or more anterior region it can have an imminent psychological and functional ill effect on the patient [12]. It has been emphasized that early diagnosis of hypodontia can result in minimal functional, psychological, and esthetic complications which may have to be dealt with later in life of the patient [13], [14]. Orthodontic space redistribution, fixed partial denture and implants are considered as standard treatment options for these patients which can help the patient lead a normal functional life [14], [15]. 
The current study was designed to understand the prevalence of bilateral agenesis of mandibular second premolars. The authors also have tried to suggest possible clinical management options of congenitally missing mandibular second premolars.

\section{MATERIALS AND METHODOLOGY}

This was an observational retrospective study conducted after approval from the Research and Ethics Committee. This study evaluated the prevalence of bilateral agenesis of the mandibular second premolars. Clinical examination of these patients was not possible, therefore only those OPGs which showed bilateral absence were considered to be true agenesis and were included in this study. The study also assessed the gender and arch predilection for the bilateral agenesis of these teeth. The age group of the patients of whom the OPGs were selected was between 6years to 30 years of age.

945 Orthopantamograms (OPGs) were first included out of the total 18500 OPGs available. These OPGs were selected based upon the age group and clear details like clarity of OPG, date of OPG taken and gender of patient. The health files of these patients which are electronically preserved in the university were evaluated to exclude patients with syndromes and also those patients were excluded if they had undergone extractions of the premolars for any reason. Once these 945 OPGs were included then these OPGs were searched for bilateral agenesis.

\section{A. Statistical Analysis}

Data observed in this study was described using descriptive statistical analysis. To evaluate the frequency of agenesis between the sexes (males/females), and chi-square statistical test was applied, the level of significance was set at $\mathrm{P}<0.05$.

\section{RESULTS}

Bilateral agenesis was found in 85 OPGs out of the 945 OPGs. $10.5 \%$ showed bilateral agenesis or congenitally missing mandibular second premolars (The remaining OPGs of these 85 showed bilateral agenesis of maxillary lateral incisors and third molars) (Fig. 1).

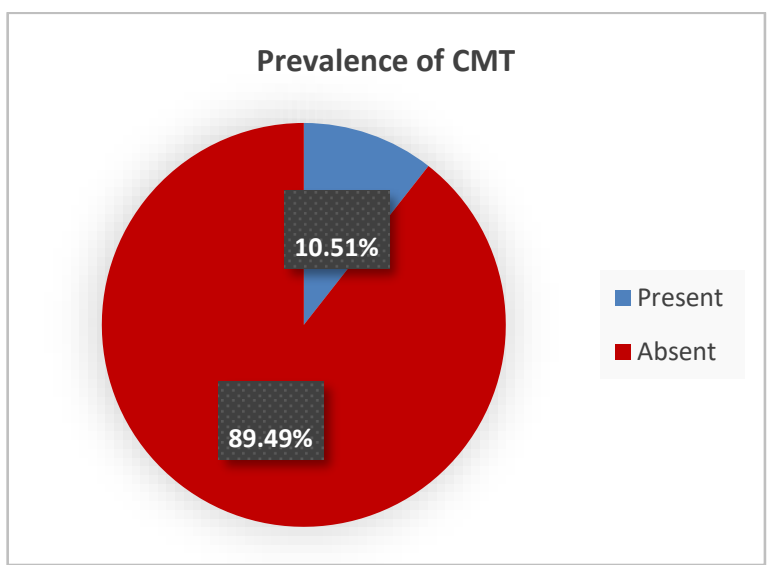

Fig. 1. Prevalence percentage of bilateral agenesis of mandibular second premolar.
The prevalence of bilateral agenesis or congenitally missing teeth was seen more in males $(55.55 \%)$ than females $(44.44 \%)$ (Table I), the results were however not statistically significant $\left(\mathrm{X}^{2}=0.84, \mathrm{P}=0.30\right)$.

TABLE I: PREVALENCE OF BILATERAL AgENESIS OR CONGENITALLY Missing TEETH BETWEEN MALES AND FEMALES

\begin{tabular}{cccccc}
\hline Teeth & Total & Male & Female & $\begin{array}{c}\mathrm{X}^{2}(\mathrm{Chi} \\
\text { Square })\end{array}$ & $\begin{array}{c}\text { Sig p } \\
\text { Value }\end{array}$ \\
\hline $\begin{array}{c}\text { Mandibular } \\
\text { Second } \\
\text { Premolars }\end{array}$ & $100 \%$ & $55.55 \%$ & $44.44 \%$ & 0.84 & 0.30 \\
\hline
\end{tabular}

\section{DISCUSSION}

Studies have suggested agenesis to have a prevalence rate of $25 \%$ amongst the general population making it one of the commonest dental anomalies in humans [9]. This anomaly is associated with other conditions like crowding and delayed eruption [10]. The permanent dentition is more affected with agenesis when compared to the primary dentition [11]. In the present study the prevalence rate of bilateral agenesis of mandibular second premolars have been assessed. A tooth was defined as to be with agenesis if there were no signs of mineralization of the crown evident on the OPG and also a second level of confirmation was done using the electronic files of the patients confirming if the premolars were not extracted for any reason.

In this retrospective study a total of 945 OPGs were initially included of which 85 OPGs showed evidence of bilateral agenesis or congenitally absent teeth including third molars. Out of these 85 OPGs $10.5 \%$ reflected agenesis of mandibular second premolars.

\section{A. Gender Predilection}

The prevalence for bilateral agenesis of mandibular second premolars was seen more in males $(55.55 \%)$ than females $(44.44 \%)$ (Table I). There are studies which have commonly shown an increased rate of prevalence of agenesis in females when compared to males [13], [14]. Other studies have shown similar results like the present study and have shown higher incidence rates in males when compared to females, these studies were reported from Iran, Australia, and Finland [12], [18], [19]. It has been shown in studies that the prevalence of hypodontia is usually higher in females [10]. However, when the literature is explored there is not much evidence or reasoning as to why the prevalence is higher or lower in either gender though genetics and hereditary factors have been attributed as a strong reasoning factor [20].

The agenesis of mandibular second premolars beholds significant clinical management challenges to the clinicians. The space created by the agenesis of these teeth should be ideally treated by either space redistribution, implants, orthodontic space closure or implants. The management of edentulous spaces involving these teeth must be done appropriately by the orthodontist.

\section{B. Clinical Management of Agenesis of Mandibular Second Premolars}

The clinical management of the agenesis of mandibular second premolars can be divided into the following scenarios. 
1. When there is a space corresponding to the second premolar as a result of the exfoliation of the primary second molar.

2. When there is no space corresponding to the second premolar., i.e., the permanent first molars have drifted mesially and space is lost.

3. When the second deciduous molar is retained.

C. When There is a Space or No Space Corresponding to the Second Premolar as a Result of the Exfoliation of the Primary Second Molar

Whenever there is a space to deal with there are two scenarios, one is a decision to leave the space as such open until a final restoration is decided upon later in life. If it is decided to do so, then we should ensure that there is enough space for a final restoration. Also, it is necessary to ensure the health of the alveolar ridge so as to take upon a dental implant as the final restoration [14], [17].

If it is decided upon by the orthodontist to close the space, then the orthodontist should ensure that the management will not interfere with normal occlusion or should not create an unfavorable adjustment which can harm the facial profile. It is to be understood whatever decisions are made should be in the best interest of the patient as the patient has to survive this for lifetime. Therefore, appropriate decision making by the orthodontist as early as possible is recommended [17].

\section{When the Second Deciduous Molar is Retained}

Whenever a second deciduous molar is retained there are two possibilities either extract the second deciduous molar or then go for a space maintainer until it is time for a final restoration which in all probability is a dental implant. Another option is to maintain the second deciduous molar with some modifications. If it is decided to extract the second deciduous molar and then go for space management and then followed by dental implant when it is time, it needs to be remembered that after the extraction, the alveolar bone goes through resorption. In the cases of second deciduous molars, it has been seen that the resorption mainly occurs towards the buccal side when compared to the lingual side and then the implant will have to be placed more lingually than bucally. This is a significant factor which will need to be considered [17]. However, if it is decided to retain the second deciduous molar then it is suggested that the mesiodistal width of the second deciduous molar be reduced and brought to the same size of a premolar and thereby allowing space redistribution and closure by the drifting of adjacent teeth. However, factors which need to be considered if this is the chosen path of treatment include the consideration that the pulp of the primary tooth should not be disturbed else a pulpectomy should be considered before reduction of the mesiodistal width [17]. If the pulp is affirmatively not going to be disturbed, then the tooth after reduction can be protected by a layer of acid etch composite restoration. Also, another factor needs to be understood is because of the divergent nature of the roots of the deciduous second molar the adjacent teeth may not show drifting as desired [18].

The authors of the present study believe that there should be increased number of samples included to give formidable results and also to understand the gender predilection clearly regarding the prevalence rates. Studies should also be done on a larger scale to understand the genetics behind agenesis.

However, the management options of agenesis of second premolar are equally challenging and exciting at the same time. It has to be understood that there are no straightforward methods in the management of agenesis of the premolars and the dentist has to be more flexible, accommodative and actually innovative in understanding the situation comprehensively and deal accordingly. Evidence based dental practice would be the best way forward.

\section{CONCLUSIONS}

In the present study we found that:

1. The prevalence rate of bilateral agenesis or congenitally missing mandibular second premolars is at $10.5 \%$.

2. Clinical management options of congenitally missing mandibular second premolars need to be based on evidence based dental practice.

\section{REFERENCES}

[1] Moyers RE, Riolo ML. Early treatment. In: Moyers RE, editor Handbook of orthodontics, 4th ed. Chicago: Year Book Medical Publishers; 1988. p. 348-53.

[2] Silva MR. Radiographic assessment of congenitally missing teeth in orthodontic patients. Int J Paediatr Dent 2003; 13:112-6.

[3] Rakhshan V. Meta-analysis and systemic review of factors biasing the observed prevalence of congenitally missing teeth in permanent dentition excluding third molars. Prog Orthod. 2013; 14:33.

[4] Bural C, Oztas E, Ozturk S, Bayraktar G. Multidisciplinary treatment of nonsyndromic oligodontia. Eur J Dent. 2012; 6:218-6.

[5] Closs LQ, Weissbluth MF, Nakamura E, Hermann FP. Esthetic and functional rehabilitation for oligodontia in the mixed dentition: case report. J Dent Child. 2012; 79(3):193-6.

[6] Parkin N, Elcock C, Smith RN, Griffin RC, Brook AH. The aetiology of hypodontia: the prevalence, severity and location of hypodontia within families. Arch Oral Biol. 2009;54(1):S52-6.

[7] Lo Muzio L, Mignogna MD, Bucci P, Sorrentino F. Indagine statistica sull'incidenza delle agenesie in un campione di 1529 soggetti. Minerva Stomatol. 1989;28:1045-51

[8] Laganà G, Venza N, Borzabadi-Farahani A, Fabi F, Danesi C, Cozza P. Dental anomalies: prevalence and associations between them in a large sample of non-orthodontic subjects, a cross-sectional study. BMC Oral Health. 2017; 17:62.

[9] Kokich VO Jr, Kinzer GA. Managing congenitally missing lateral incisors Part II: Tooth-supported restorations. J. Esthet Restor Dent 2005; 17(2):76-84.

[10] Muhamad Abu-Hussein et al.: Prevalence of Missing Lateral Incisor Agenesis in an Orthodontic Arabs Population in Israel (Arab48). International Journal of Public Health Research 2015; 3(3):101-107.

[11] Larmour C, Mossey PA, Thind BS, Forgie AH, Stirrups DR Hypodontia - a retrospective review of prevalence and etiology. Part I. Quintessence Int. 2005; 36(4):263-70.

[12] Vahid-Dastjerdi E, Borzabadi-Farahani A, Mahdian M, Amini N Non-syndromic hypodontia in an Iranian orthodontic population. J of Oral Science. 2010; 52(3):455-461.

[13] Polder BJ, Van't Hof MA, Van der Linden FP, Kuijpers-Jagtman AM. A meta-analysis of the prevalence of dental agenesis of permanent teeth. Community Dent Oral Epidemiol 2004; 32:217-26.

[14] Kokich VG, Kokich VO. Congenitally missing mandibular second premolars: Clinical options. Am J Orthod Dentofacial Orthop 2006; 130:437-44.

[15] Kokich VO Jr, Kinzer GA. Managing congenitally missing lateral incisors Part II: Tooth-supported restorations. J. Esthet Restor Dent 2005; 17(2):76-84.

[16] Kennedy D. Missing Second Premolars: Can Early Treatment Make a Difference? Spring Vancouver Canada: PCSO Bulletin; 2010. p. 2933.

[17] Spear F, Mathews D, Kokich V. Interdisciplinary management of single-tooth implants. Semin Orthod 1997; 3:45-72. 
[18] Habsha E. The incidence of pulpal complications and loss of vitality subsequent to full crown restorations. Ont Dent 1998; 75: 19-21.

[19] Ostler M, Kokich V. Alveolar ridge changes in patients congenitally missing mandibular second molars. J Prosthet Dent 1994; 71:144-9.

[20] Kokich V, Spear F. Guidelines for managing the orthodontic restorative patient. Semin Orthod 1997; 3:3-20. 